\title{
Near-shore sediment dynamics computation under the combined effects of waves and currents
}

\author{
J.S. Antunes Do Carmo*, F.J. Seabra-Santos \\ IMAR, Department of Civil Engineering, University of Coimbra, Pólo II-Pinhal de Marrocos, 3030-290 Coimbra, Portugal
}

Received 30 May 2001; revised 1 August 2001; accepted 8 August 2001

\begin{abstract}
An integrated computational structure for non-cohesive sediment-transport and bed-level changes in near-shore regions has been developed. It is basically composed of: (1) three hydrodynamic sub-models; (2) a dynamic equation for the sediment transport (of the Bailardtype); and (3) an extended sediment balance equation. A shallow-water approximation, or Saint-Venant-type model, is utilized for the computation and up-to-date field currents, initially and after each characteristic computational period. A Berkhoff-type wave model allows us to determine the wave characteristics in deep water and intermediate water conditions. These computations make it possible to define a smaller modeling area for a non-linear wave-current model of the Boussinesq-type, including breaking waves, friction effects and improved dispersion wave characteristics. Bed topography is updated after each wave period, or a multiple of this, called computational sedimentary period. Applicability of the computational structure is confirmed through laboratory experiments. Practical results of a real-world application obtained around the S. Lourenço fortification, Tagus estuary (Portugal), with the intention of preventing the destruction of the Bugio lighthouse, are shown. (C) 2002 Elsevier Science Ltd. All rights reserved.
\end{abstract}

Keywords: Deep-water; Shallow-water; Wave-current interaction; Extended Boussinesq equations; Sediment-transport model

\section{Introduction}

Shallow coastal areas are extremely dynamic regions where the fluid motions associated with both surface waves and currents interact with the bottom sediments. As pointed out by Grant and Madsen [1] and Asano and Iwagaki [2], among others, in these regions the wave and current motions cannot be treated separately and then superposed. Indeed, the fluid dynamics of the combined wave and current motions and, as a consequence, the response of the movable seabed are significantly altered from that expected for a linear superposition of a pure wave motion with a pure current.

Moreover, the prediction of the waves effect on sediment transport in shallow water and in intermediate depth is still frequently restricted to monochromatic and unidirectional wave models. In real shallow water conditions, the nonlinear process of sediment transport responds in a rather different way to the idealized regular wave case. Therefore, in these regions, the wave non-linearity and the wave-

\footnotetext{
* Corresponding author. Tel.: +351-239-797-153; fax: +351-239-797152.

E-mail address: jsacarmo@dec.uc.pt (J.S. Antunes Do Carmo).
}

current interaction become important factors to be considered.

Other effects must be also considered, such as those resulting from the bottom friction and the wave breaking process, the latter being also responsible for producing littoral currents.

Actually, the problem must be treated considering all resulting complex interactions effects their entirety. Of course, all other relevant near-shore processes, such as shoaling, reflection, refraction, diffraction and decomposition must also be taken into account.

The purpose of this paper is to present an integrated computational structure of near-shore processes; it is mainly composed by:

1. A refraction-diffraction model, based on linear theory, to relate the agitation characteristics from different points situated in deep or intermediate water conditions, and namely for the transportation of information relative to wave characteristics from offshore to the near-shore region. For large regions, this is done in two phases: firstly refraction planes are obtained and then results are improved using Berhoff's equation. This procedure provides initial and boundary conditions for the near field simulation;

2. A current model, based on the classical Saint-Venant or 
shallow-water equations, taking into account the radiation stresses, for the computation of the current field;

3. A wave-current model based on an extended form of modified Boussinesq equations, applicable to the computation of the wave deformation due to combined effects of shoaling, reflection, refraction, diffraction, breaking waves and wave-current interactions from intermediate to very shallow water conditions $(k h \leq 1)$ over slowly varying slopes of the bed;

4. A sediment-transport model based on a Bailard-type dynamic formula, to compute the bed load and suspended transport rates, combined with an extended sediment balance equation to compute the bed evolution.

Theoretical aspects are treated in the next section. Numerical computations, together with a comparison with experimental data and a real-world applications will come afterwards.

\section{Formulation}

\subsection{Wave propagation from offshore to the near field}

The linear approximation to the sea surface elevation caused by the propagation of a small amplitude time-harmonic wave can be obtained as the solution of the extended mild-slope equation:

$$
\frac{\partial}{\partial x}\left(c c_{g} \frac{\partial \bar{\eta}}{\partial x}\right)+\frac{\partial}{\partial y}\left(c c_{g} \frac{\partial \bar{\eta}}{\partial y}\right)+\frac{c_{g}}{c} w^{2} \bar{\eta}+i w W \bar{\eta}=0
$$

where $\bar{\eta}=\bar{\eta}(x, y)$ is a complex function including both amplitude and phase of the surface elevation; $c$ and $c_{g}$ are the Airy's phase celerity and group celerity, respectively; and $w$ is the wave angular frequency. $W$ is the ratio between the energy dissipation per surface unit area per unit time and the energy per unit area. A linear approximation for the velocity field is derived as:

$u=-i \frac{g}{w} \frac{\partial \bar{\eta}}{\partial x} ; \quad v=-i \frac{g}{w} \frac{\partial \bar{\eta}}{\partial y}$

As discussed earlier, for large regions this procedure may be considered as an improvement to the method of refraction planes. Besides, refraction planes are also useful to define impermeable boundaries $(u \cdot n=0)$ for the Berkhoff model.

Refraction planes are obtained from a linear wave propagation model, considering the wave direction $(\theta)$ and the wave celerity $(C)$ as dependent variables. In intrinsic coordinates ( $s$ and $n$ ), such that $s$ is in the wave direction and $n$ normal to it, defined as:

$x=s \cos \theta-n \sin \theta$

$y=s \sin \theta+n \cos \theta$

the following expression yields [3]:

$\frac{\partial \theta}{\partial s}=\frac{1}{k} \frac{\partial k}{\partial n}=-\frac{1}{C} \frac{\partial C}{\partial n}$

where $k$ is the wave number.
This equation relates the curvature of the wave ray to the logarithmic derivative of $C$ normal to the wave direction. It can be efficiently solved by an explicit finite-difference scheme applied to the two initial phase lines (i.e. crests) of a wave propagating in deep water conditions. A refraction plane is presented later.

It is necessary to establish a procedure when the sea surface characteristics are known (from measured records) at some points near the coast but far from the area of interest. In these situations, the corresponding wave characteristics in these points are calculated in two stages.

In the first stage, the characteristics of the sea surface are carried to the open sea by inversion of the natural propagation of the wave, considering an initial phase line of reduced extension. In the second stage, a rectilinear phase line of long extension may be considered, assuming deep water, and the wave is then propagated from the open sea to the region of interest, where the information is passed to the non-linear model.

This procedure was utilized by the authors to obtain the sea surface characteristics at the entrance of the port of Figueira da Foz (Portugal). These characteristics were then used as boundary conditions to a non-linear Serre wave model utilized to compute the results presented in Antunes do Carmo et al. [4].

As discussed in Avilez-Valente et al. [5], when the domain length is large in the wave propagation direction, Berkoff's linear solution may be used as an initial condition for the non-linear wave-current model of the Boussinesqtype. This numerical procedure allows a faster convergence with considerable saving in computing time.

\subsection{Current computations}

The current model is based on the classical Saint-Venant or shallow-water equations, and provides stationary current field (relative to the time scale of the wave simulation), over which waves propagate.

Taking into account the radiation stresses $\left(S_{x x}, S_{x y}, S_{y x}\right.$ and $S_{y y}$ ) as defined by Longuet-Higgins and Stewart [6], the governing equations for the current field computation are as follows:

$$
\begin{aligned}
& \frac{\partial h}{\partial t}+\frac{\partial\left(h u_{c}\right)}{\partial x}+\frac{\partial\left(h v_{c}\right)}{\partial y}=0 \\
& \frac{\partial u_{c}}{\partial t}+u_{c} \frac{\partial u_{c}}{\partial x}+v_{c} \frac{\partial u_{c}}{\partial y}+g \frac{\partial(h+\xi)}{\partial x}+\frac{1}{\rho h}\left(\frac{\partial S_{x x}}{\partial x}+\frac{\partial S_{x y}}{\partial y}\right) \\
& \quad-\frac{\tau_{s_{x}}}{\rho h}+\frac{\tau_{b_{x}}}{\rho h}=0 \\
& \frac{\partial v_{c}}{\partial t}+u_{c} \frac{\partial v_{c}}{\partial x}+v_{c} \frac{\partial v_{c}}{\partial y}+g \frac{\partial(h+\xi)}{\partial y}+\frac{1}{\rho h}\left(\frac{\partial S_{y x}}{\partial x}+\frac{\partial S_{y y}}{\partial y}\right) \\
& -\frac{\tau_{s_{y}}}{\rho h}+\frac{\tau_{b_{y}}}{\rho h}=0
\end{aligned}
$$

where $\left(u_{c}, v_{c}\right)$ represent the current field; $h=H-\xi+\eta$, being $H-\xi$ and $\eta$ the still water depth and the surface 
elevation, respectively; $H$ is a constant characteristic depth, relative to a datum, $\xi$ represents bed levels, and $\eta$ represents surface variations; $\tau_{b}$ and $\tau_{s}$ represent stresses at the bottom and at the surface, respectively.

Equation system (3) is solved iteratively using a finite element method. Accordingly to this method, considering a function $p$ any of the computational variables $\left(h, u_{c}, v_{c}\right.$ and $\xi)$ this is approximated within each element by:

$p \approx \hat{p}=\sum_{i=1}^{n} N_{i} p_{i}$

where $p_{i}$ is the value of the function $p$ at the node $i$ of a generic element $\Delta^{e}, n$ is the number of nodes of the element and $N_{i}$ is the interpolation (shape) function $N$ corresponding to the $i$-node of the element $\Delta^{e}$.

The substitution of $\hat{p}$ in any equation $(k)$ of the system (3) ( $k=1,2,3)$ generates a residual $R_{k}$. The weighted residual technique utilized here requires the 'orthogonality' of the residuals $R_{k}$ to a set of weighting functions $W_{i}$, i.e.

$\int_{\Delta^{e}} W_{i} R_{k} d \Delta^{e}=0$

where the weighting functions $W_{i}$ are functions of $N_{i}$ and its derivatives [7].

\subsection{Extended Boussinesq equations}

These equations have been derived by vertical integration of the fundamental equations relating to a three-dimensional and quasinon-rotational flow of a viscous and incompressible fluid. A complete set of these equations is presented in Antunes do Carmo et al. [4] and Antunes do Carmo [8] without current interaction, although with time-varying movable bottoms.

Another set of these equations, taking into account wavecurrent interactions, and with improved dispersive characteristics, are presented in Antunes do Carmo and SeabraSantos [7] and Antunes do Carmo [9].

With dimensional variables, taking mean quantities of the horizontal velocity components $U=\left(u_{w}+u_{c}\right)$ and $V=\left(v_{w}+v_{c}\right)$, this latter set of equations may be written as follows:

$$
\begin{aligned}
& \frac{\partial h}{\partial t}+\frac{\partial(h U)}{\partial x}+\frac{\partial(h V)}{\partial y}=0 \\
& \frac{\partial U}{\partial t}+U \frac{\partial U}{\partial x}+V \frac{\partial U}{\partial y}+g \frac{\partial(h+\xi)}{\partial x} \\
& \quad-\beta\left(\frac{\partial^{3} U}{\partial x^{2} \partial t}+\frac{\partial^{3} V}{\partial x \partial y \partial t}\right) \\
& -\beta \frac{\partial}{\partial x}\left[u_{c}\left(\frac{\partial^{2} U}{\partial x^{2}}+\frac{\partial^{2} V}{\partial x \partial y}\right)+v_{c}\left(\frac{\partial^{2} U}{\partial x \partial y}+\frac{\partial^{2} V}{\partial y^{2}}\right)\right] \\
& -\nu\left(\frac{\partial^{2} U}{\partial x^{2}}+\frac{\partial^{2} U}{\partial y^{2}}\right)-\frac{\tau_{s_{x}}}{\rho h}+\frac{\tau_{b_{x}}}{\rho h} \\
& =0
\end{aligned}
$$

$$
\begin{aligned}
& \frac{\partial V}{\partial t}+U \frac{\partial V}{\partial x}+V \frac{\partial V}{\partial y}+g \frac{\partial(h+\xi)}{\partial y} \\
& -\beta\left(\frac{\partial^{3} U}{\partial x \partial y \partial t}+\frac{\partial^{3} V}{\partial y^{2} \partial t}\right) \\
& -\beta \frac{\partial}{\partial y}\left[u_{c}\left(\frac{\partial^{2} U}{\partial x^{2}}+\frac{\partial^{2} V}{\partial x \partial y}\right)+v_{c}\left(\frac{\partial^{2} U}{\partial x \partial y}+\frac{\partial^{2} V}{\partial y^{2}}\right)\right] \\
& -\nu\left(\frac{\partial^{2} V}{\partial x^{2}}+\frac{\partial^{2} V}{\partial y^{2}}\right)-\frac{\tau_{s_{y}}}{\rho h}+\frac{\tau_{b_{y}}}{\rho h} \\
& =0
\end{aligned}
$$

where $h=H-\xi+\eta$, representing $\xi$ and $H$ bottom levels and a vertical distance, both relative to a fixed datum; $\eta$ represents surface variations;

$\beta=\frac{1}{k^{2}}\left\{\frac{k(H-\xi)}{\tan h[k(H-\xi)]}-1\right\}$,

where $k$ is the wave number; and $\nu$ is the kinematic viscosity, assumed constant.

Assuming a quadratic friction law, the $\vec{\tau}_{b} / \rho h$ term may be written as

$$
\frac{\vec{\tau}_{b}}{\rho h}=\frac{1}{2 h} f_{c w}|\vec{V}| \vec{V}
$$

where

$$
\vec{V}=(U, V) ; \quad|\vec{V}|=\sqrt{U^{2}+V^{2}} ;
$$

$f_{c w}=\frac{\left|\vec{V}_{w}\right|}{\left|\vec{V}_{w}\right|+\left|\vec{V}_{c}\right|} f_{w}+\frac{\left|\vec{V}_{c}\right|}{\left|\vec{V}_{w}\right|+\left|\vec{V}_{c}\right|} f_{c}$,

where $f_{c w}$ is the wave-current friction factor, with

$\vec{V}_{w}=\left(u_{w}, v_{w}\right), \quad \vec{V}_{c}=\left(u_{c}, v_{c}\right)$,

$f_{w}=0.00278 e^{4.65\left(\frac{\hat{a}}{k_{N}}\right)^{-022}}, \quad f_{c}=0.06\left(\log _{10} \frac{12 h}{k_{r c w}}\right)^{-2}$

and with the equivalent Nikuradse roughness length $k_{N} \cong 2.5 D_{50}$, a current-related bed-roughness coefficient $k_{\text {rcw }} \cong 3 D_{90}$ (bed-forms are not taken into account),

$\hat{a}=\frac{\sqrt{2} V_{r m s} T}{2 \pi}, \quad V_{r m s}=\sqrt{\frac{1}{N} \sum_{i=1}^{N} V_{i n}^{2}}$,

being $V_{\text {in }}$ signal input velocities, and $N=T / \Delta t$ with $T$ the characteristic wave period.

As can be seen in Antunes do Carmo and Seabra-Santos [7], a general expression for the energy dissipation term due to the wave breaking process is

$$
\frac{\vec{\tau}_{s}}{\rho h}=-\vec{\nu}_{t}\left(\frac{\partial^{2} \vec{V}_{w}}{\partial x^{2}}+\frac{\partial^{2} \vec{V}_{w}}{\partial y^{2}}\right)
$$


where $\vec{\nu}_{t}$ is a parametric expression representing the viscosity of generated turbulence.

Equation system (4) is solved using a finite element method, after grouping the $(U, V)$ derivatives in time and third spatial derivatives in two equations. For details about the solution procedure and numerical implementation, refer to Refs. [4,7].

The grid can be the same for both the current and the wave-current computations. However, computational requirements for the wave-current model are very restrictive. Strictly, the element side should be lower than the local depth, and a minimum of about 20-25 elements per wave-length is needed. In practice, it could be more efficient to use two grids. The current velocity field is obtained in a computational coarse grid and results are then interpolated to a more re-fined grid, which is used to run the Boussinesqtype model.

Several regular and highly irregular quadrilateral grids have been used to the present, and the computational structure behaves well. The numerical procedure requires a time increment $(\Delta t)$ determined according to the Courant-Friedrich-Lewy condition, with the Courant number always less than one over the whole domain (see reference bibliography for details).

\subsection{Sediment transport and mobile bed evolution}

\subsubsection{Improved Bailard dynamic equation}

Based on the pioneering works of Bagnold [10,11], the energetics approach was extended for alongshore and crossshore bed load sediment transport in a parallel development to Bowen [12], Bailard and Inman [13] and Bailard [14] extended this theory to account for suspended load. The resulting vector form of the total sediment transport rate $\left\langle\vec{q}_{s t}\right\rangle$ is,

$$
\begin{gathered}
\left\langle\vec{q}_{s t}\right\rangle=\frac{c_{f}}{g(s-1)}\left\{\frac{\epsilon_{b}}{\tan \phi}\left[\left\langle\left|\vec{V}_{b}^{2}\right| \vec{V}_{b}\right\rangle-\frac{\tan \alpha}{\tan \phi}\left\langle\left|\vec{V}_{b}^{3}\right|\right\rangle i\right]\right. \\
\left.+\frac{\epsilon_{s}}{w_{f}}\left[\left\langle\left|\vec{V}_{b}^{3}\right| \vec{V}_{b}\right\rangle-\frac{\epsilon_{s}}{w_{f}} \tan \alpha\left\langle\left|\vec{V}_{b}^{5}\right|\right\rangle i\right]\right\}
\end{gathered}
$$

where $\langle\cdots\rangle$ denotes average quantities; $c_{f}$ is a friction coefficient; $\tan \alpha$ is the slope of the bed; $\phi$ is the internal angle of friction of the sediment; $w_{f}$ represents the fall velocity of the sediment. The total near-bed fluid velocity is $\vec{V}_{b}=\tilde{V}_{b}+\bar{V}_{b}$, with $\tilde{V}_{b}$ the oscillatory velocity component and $\bar{V}_{b}$ the steady current component.

Considering in Eq. (5) the vertical mean horizontal fluid velocity $\vec{V}$, resulting from equations system (4), and the friction coefficient $c_{f}=\sqrt{0.5 f_{c w}}$, from comparisons of numerical computations with experimental data, the efficiencies $\epsilon_{b}$ and $\epsilon_{s}$ seem to have values in the intervals:

$\epsilon_{b} \approx 0.05-0.25$

$\epsilon_{s} \approx 0.01-0.03$
As it is well reported in the literature [15,16], Eq. (5) constitutes a good model when sediments grain diameter is equal to or greater than about $0.15-0.20 \mathrm{~mm}$, which is normally the case dealing with non-cohesive sediments in near-shore coastal regions.

A threshold condition for sheet flow based on the Shields diagram limit, with $V_{*_{0}}=\sqrt{\tau_{0} / \rho}$ and $\tau_{0} \cong 0.05 \rho(s-1) D_{50}$, taking into account the slope of the bed, is given by Eq. (6) below [9]:

$\sqrt{0.5 f_{c w}} \vec{V} \cong \vec{V}_{*}>\vec{V}_{*} \sqrt{\cos \alpha_{s} \cos \alpha_{t}\left(1-\frac{\tan ^{2} \alpha_{t}}{\tan ^{2} \phi}\right)^{2}+\frac{\sin \alpha_{s}}{\tan \phi}}$

where $\vec{V}_{*_{0}}$ is the critical friction velocity in horizontal conditions; $\tan \alpha_{s}$ is the local bed slope in the flow direction. Considering $\tan \alpha_{n}$ the bed slope in normal direction of the flow, $\alpha_{t}$ is the angle that satisfies $\tan \alpha_{t}=\cos \alpha_{s} \tan \alpha_{n}$.

So, in order to have transport, condition (6) should be satisfied.

As shown in the next section, the Bailard dynamic Eq. (5) together with Eq. (6) form the basis of our sedimentary model.

\subsubsection{Seabed evolution: sediment balance equation}

As included in the Bailard formula, slope effects in the bed load as well as the suspended load part are very important in coastal profile evolution. Due to this effect, the sediment will be transported more easily downslope than upslope, which has a stabilizing effect on the profile evolution.

Another way to take the slope effect into account is to adjust the transport vector as described in Horikawa [17]. Representing $q_{b}$ and $q_{s}$ the bed load and suspended load, respectively, the following expressions can be written:

- for bed load:

$$
\left\{\begin{array}{l}
q_{b_{x}}=q_{b 0_{x}}-\epsilon_{b_{x}}\left|q_{b 0_{x}}\right| \frac{\partial \xi}{\partial x} \\
q_{b_{y}}=q_{b 0_{y}}-\epsilon_{b_{y}}\left|q_{b 0_{y}}\right| \frac{\partial \xi}{\partial y}
\end{array}\right.
$$

- for suspended load:

$$
\left\{\begin{array}{l}
q_{s_{x}}=q_{s 0_{x}}-\epsilon_{s_{x}}\left|q_{s 0_{x}}\right| \frac{\partial \xi}{\partial x} \\
q_{s_{y}}=q_{s 0_{y}}-\epsilon_{s_{y}}\left|q_{s 0_{y}}\right| \frac{\partial \xi}{\partial y}
\end{array}\right.
$$

As total load formulae in each direction are: $q_{s t_{x}}=q_{b_{x}}+q_{s_{x}}$ and $q_{s t_{y}}=q_{b_{y}}+q_{s_{y}}$, we can write:

$$
\left\{\begin{array}{l}
q_{s t_{x}}=q_{b 0_{x}}+q_{s 0_{x}}-\left(\epsilon_{b_{x}}\left|q_{b 0_{x}}\right|+\epsilon_{s_{x}}\left|q_{s 0_{x}}\right|\right) \frac{\partial \xi}{\partial x} \\
q_{s t_{y}}=q_{b 0_{y}}+q_{s 0_{y}}-\left(\epsilon_{b_{y}}\left|q_{b 0_{y}}\right|+\epsilon_{s_{y}}\left|q_{s 0_{y}}\right|\right) \frac{\partial \xi}{\partial y}
\end{array}\right.
$$


Substituting these transport vector components (7) into the sediment balance equation, assuming some simplifications, yields the following equation:

$$
\begin{aligned}
& (1-\lambda) \frac{\partial \xi}{\partial t}+\frac{\partial\left\langle q_{b 0}+q_{s 0}\right\rangle_{x}}{\partial x}+\frac{\partial\left\langle q_{b 0}+q_{s 0}\right\rangle_{y}}{\partial y} \\
& -\left\langle\epsilon_{b}\left|q_{b 0}\right|+\epsilon_{s}\left|q_{s 0}\right|\right\rangle_{x} \frac{\partial^{2} \xi}{\partial x^{2}}-\left\langle\epsilon_{b}\left|q_{b 0}\right|+\epsilon_{s}\left|q_{s 0}\right|\right\rangle_{y} \frac{\partial^{2} \xi}{\partial y^{2}} \\
& \quad=0
\end{aligned}
$$

where $\lambda$ represents the porosity of the bed (in general $\lambda \cong 0.35$ ) and $\xi$ is a vertical distance relative to a fixed datum.

According to Eq. (5), it can be seen that:

$$
\begin{aligned}
& \left\langle q_{b 0}\right\rangle_{x}=\frac{C_{f}}{g(s-1)} \frac{\epsilon_{b}}{\tan \phi}\left\langle|u|^{2} u\right\rangle ; \\
& \left\langle q_{s 0}\right\rangle_{x}=\frac{C_{f}}{g(s-1)} \frac{\epsilon_{s}}{w_{f}}\left\langle|u|^{3} u\right\rangle ; \\
& \left\langle q_{b 0}\right\rangle_{y}=\frac{C_{f}}{g(s-1)} \frac{\epsilon_{b}}{\tan \phi}\left\langle|v|^{2} v\right\rangle ; \\
& \left\langle q_{s 0}\right\rangle_{y}=\frac{C_{f}}{g(s-1)} \frac{\epsilon_{s}}{w_{f}}\left\langle|v|^{3} v\right\rangle ; \quad \epsilon_{b_{x}}=\epsilon_{b_{y}}=\frac{1}{\tan \phi} ; \\
& \epsilon_{s x}=\epsilon_{s}\left|\frac{u}{w_{f}}\right| \quad \text { and } \quad \epsilon_{s_{y}}=\epsilon_{s}\left|\frac{v}{w_{f}}\right|
\end{aligned}
$$

For the solution of Eq. (8), a numerical procedure based on finite elements is also employed.

\section{Numerical computations}

The integrated computational structure presented above is based on depth-averaged current and wave-current modules, a sediment transport equation and a bottom evolution module based on sediment conservation; it may be considered a short to medium-term morphodynamic model, which is applicable under the following assumptions:

1. The time and length scales associated with the wave motion are normally much lower than the corresponding current scales;

2. The transport material does not influence the water motion;

3. The time scale associated with the wave motion is much lower than the time scale associated with the mobile bed evolution.

The first assumption allows the current module to be uncoupled from the wave-current model. So, the current and the wave-current velocities are computed separately. The Saint-Venant or shallow-water Eq. (3) are able to provide the current velocity field installed, at a given moment, and the wave-current model (4) is used to compute the wave propagation and breaking over this steady current.

The second and third assumptions allow the hydrodynamic model to be uncoupled from the sediment transport equations. So, the calculated hydrodynamic values are used as known in solving the extended sediment balance Eq. (8) after each characteristic computational period.

The computations sequence of the present morphodynamic model is described in the flowchart shown in Fig. 1.

Resuming, computations are performed as follows:

- the Saint-Venant or shallow-water equations model is utilized to update the current field after some wave periods (computational sedimentary period);

- the extended wave-current Boussinesq model is utilized to obtain hydrodynamic characteristics, namely depths, mean velocities and stresses on the bed during each current-wave computational period;

- bed levels are updated after each computational sedimen-

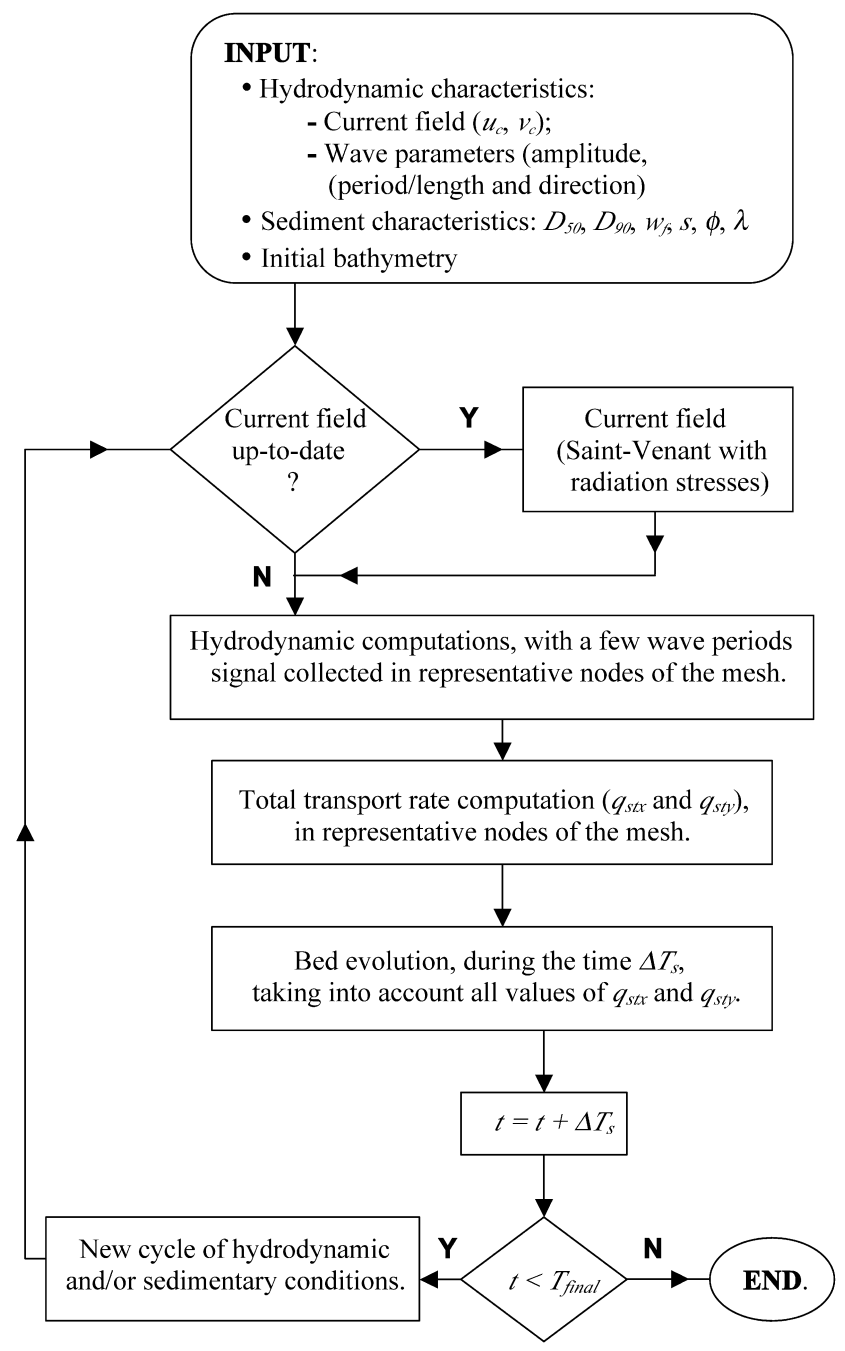

Fig. 1. General formulation of the present morphodynamic model. 
tary period according to the extended sediment balance transport model results.

It is important to note that both the computational sedimentary period and the current-wave computational period are strongly dependent on the problem characteristics. Depending on the bed level changes, the current-wave computational period will be normally much less than the period to update bed levels and the current (sedimentary period). A sub-multiple of the smallest period will be taken as the time step for the extended sediment balance computations along the bigger one, assuming unchanged current-wave characteristics.

\section{Experimental validation of the integrated computational structure}

In order to calibrate the integrated computational structure presented above for non-cohesive sediment transport, several sets of experiments have been performed by the authors. One of these experiments is presented in this section.

This experiment concerns the evolution of a sand dune under combined effects of a wave and a current. It is performed within a $7.5 \mathrm{~m}$ long by $0.30 \mathrm{~m}$ wide rectangular channel. The bottom was formed by sand with grain diameters between 0.25 and $0.30 \mathrm{~mm}$ (mean diameter, $D_{50}=0.27 \mathrm{~mm}$ ).

The initial elevation of the dune was given by

$\xi=\xi_{0} \sin ^{2}\left[\frac{\pi}{3}\left(x-x_{0}\right)\right]$

with $\xi_{0}=6.2 \mathrm{~cm}$ and $x_{0}=2 \mathrm{~m}$.

The current was generated by a centrifugal pump, with a mean velocity of $18 \mathrm{~cm} / \mathrm{s}$ and $0.20 \mathrm{~m}$ depth outside the dune.

A plunging piston located at the beginning of the channel, section $x=0$, has generated a surface wave of $1.28 \mathrm{~s}$ period and $3.8 \mathrm{~cm}$ height, whose form was captured by gauges located at four positions. The signal at the first gauge $(x=1.60 \mathrm{~m})$ was used as wave boundary condition for the hydrodynamic numerical model.

In this case, current field and bed levels were updated after each 24 wave periods (computational sedimentary period). A comparison between numerical results and experimental data $3 \mathrm{~h}$ after the beginning of the wave motion is presented in Fig. 2.

It is evident that the integration of this hydrodynamic with a mobile bed leads to small-scale rhythmic features called ripples. These bed forms have a major influence on the sediment transport, via the apparent bed roughness and the associated turbulence characteristics, and via the sediment transport mechanism. Having this in mind, general results could be considered satisfactory.

Other comparisons with laboratory experiments, such as the propagation of a wave over a steady current in a channel with irregular geometry, and the propagation and breaking of a wave over a varying-depth movable beach $[9,16]$, have been shown to be good approximations; as good as can be expected in sedimentary dynamics.

As experimental data is necessary to calibrate and validate numerical models, we present in Table 1 initial and measured bottom results 3 and $6 \mathrm{~h}$ after the beginning of the wave motion. It is important to note that current alone is not able to produce sand movement.

\section{Real-world application}

\subsection{The S. Lourenço fortification-Tagus estuary (Portugal)}

With the intention of preventing the destruction of the S. Lourenço fortification (or Bugio lighthouse), an initial protective structure was designed consisting of two breakwaters, topped at $+4 \mathrm{~m}(\mathrm{ZH})$, forming a quadrant opened to the West occupied by an artificial triangular horizontal

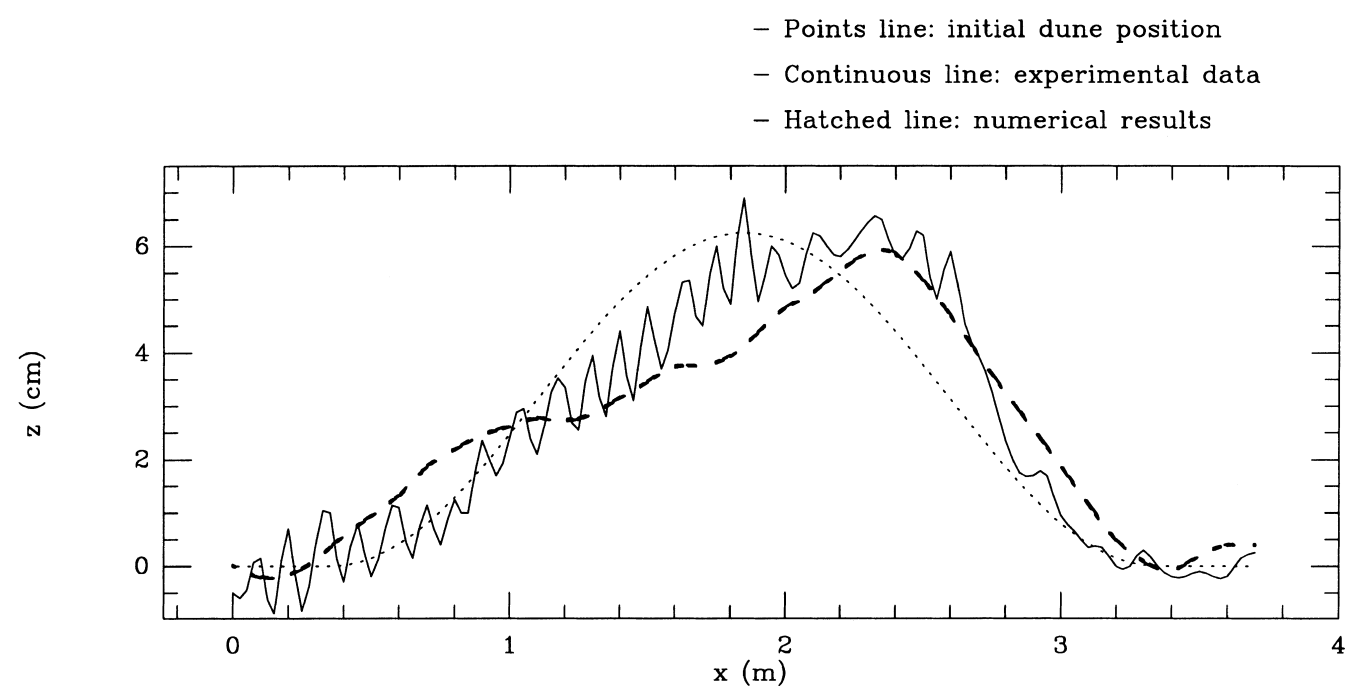

Fig. 2. Bed levels: comparisons between numerical results and experimental data ( $3 \mathrm{~h})$. 
Table 1

Initial and measured bottom results 3 and $6 \mathrm{~h}$ after the beginning of the wave motion

\begin{tabular}{|c|c|c|c|}
\hline$X_{i}(\mathrm{~m})$ & Initial (cm) & Measured (3 h) & Measured (6 h) \\
\hline 0.000 & 0.000 & -0.500 & -1.700 \\
\hline 0.050 & 0.000 & -0.450 & -0.300 \\
\hline 0.100 & 0.000 & 0.150 & -1.850 \\
\hline 0.150 & 0.000 & -0.900 & -1.000 \\
\hline 0.200 & 0.000 & 0.700 & -0.200 \\
\hline 0.250 & 0.000 & -0.850 & -0.750 \\
\hline 0.300 & 0.000 & 0.450 & -0.100 \\
\hline 0.350 & 0.000 & 1.000 & 1.000 \\
\hline 0.400 & 0.017 & -0.300 & 0.100 \\
\hline 0.450 & 0.068 & 0.800 & 0.900 \\
\hline 0.500 & 0.153 & -0.200 & 0.400 \\
\hline 0.550 & 0.270 & 0.700 & -0.100 \\
\hline 0.600 & 0.419 & 1.100 & 1.000 \\
\hline 0.650 & 0.597 & 0.150 & -0.050 \\
\hline 0.700 & 0.803 & 1.150 & 1.050 \\
\hline 0.750 & 1.034 & 0.400 & 1.050 \\
\hline 0.800 & 1.288 & 1.250 & 0.400 \\
\hline 0.850 & 1.563 & 1.000 & 1.700 \\
\hline 0.900 & 1.854 & 2.350 & 0.950 \\
\hline 0.950 & 2.159 & 1.700 & 1.850 \\
\hline 1.000 & 2.475 & 2.400 & 1.450 \\
\hline 1.050 & 2.798 & 2.950 & 2.650 \\
\hline 1.100 & 3.125 & 2.100 & 2.350 \\
\hline 1.150 & 3.452 & 3.250 & 2.300 \\
\hline 1.200 & 3.775 & 3.350 & 3.350 \\
\hline 1.250 & 4.091 & 2.550 & 3.100 \\
\hline 1.300 & 4.396 & 3.950 & 3.300 \\
\hline 1.350 & 4.687 & 2.800 & 3.150 \\
\hline 1.400 & 4.962 & 4.400 & 3.250 \\
\hline 1.450 & 5.216 & 3.100 & 3.350 \\
\hline 1.500 & 5.447 & 4.850 & 2.650 \\
\hline 1.550 & 5.653 & 3.700 & 3.700 \\
\hline 1.600 & 5.831 & 4.750 & 3.750 \\
\hline 1.650 & 5.980 & 5.350 & 3.000 \\
\hline 1.700 & 6.097 & 4.500 & 4.800 \\
\hline 1.750 & 6.182 & 6.000 & 3.600 \\
\hline 1.800 & 6.233 & 4.900 & 5.350 \\
\hline 1.850 & 6.250 & 6.900 & 3.900 \\
\hline 1.900 & 6.233 & 4.950 & 5.350 \\
\hline 1.950 & 6.182 & 6.000 & 5.950 \\
\hline 2.000 & 6.097 & 5.450 & 4.050 \\
\hline 2.050 & 5.980 & 5.300 & 5.300 \\
\hline 2.100 & 5.831 & 6.250 & - \\
\hline 2.150 & 5.653 & 6.000 & - \\
\hline 2.200 & 5.447 & 5.800 & - \\
\hline 2.250 & 5.216 & 6.100 & - \\
\hline 2.300 & 4.962 & 6.450 & - \\
\hline 2.350 & 4.688 & 6.500 & 4.700 \\
\hline 2.400 & 4.396 & 5.800 & 6.050 \\
\hline 2.450 & 4.091 & 5.950 & 5.100 \\
\hline 2.500 & 3.775 & 6.200 & 6.450 \\
\hline 2.550 & 3.452 & 5.000 & 5.550 \\
\hline 2.600 & 3.125 & 5.900 & 6.400 \\
\hline 2.650 & 2.798 & 4.550 & 5.850 \\
\hline 2.700 & 2.475 & 3.950 & 5.800 \\
\hline 2.750 & 2.159 & 3.250 & 6.850 \\
\hline 2.800 & 1.854 & 2.350 & 5.600 \\
\hline 2.850 & 1.563 & 1.750 & 5.800 \\
\hline 2.900 & 1.288 & 1.700 & 3.850 \\
\hline 2.950 & 1.034 & 1.700 & 4.800 \\
\hline 3.000 & 0.803 & 0.950 & 3.000 \\
\hline
\end{tabular}

Table 1 (continued)

\begin{tabular}{llcr}
\hline$X_{i}(\mathrm{~m})$ & Initial $(\mathrm{cm})$ & Measured $(3 \mathrm{~h})$ & Measured $(6 \mathrm{~h})$ \\
\hline 3.050 & 0.597 & 0.650 & 2.050 \\
3.100 & 0.419 & 0.350 & 1.000 \\
3.150 & 0.270 & 0.350 & 0.850 \\
3.200 & 0.153 & 0.000 & 0.550 \\
3.250 & 0.068 & 0.000 & 0.250 \\
3.300 & 0.017 & 0.300 & 0.550 \\
3.350 & 0.000 & 0.000 & -0.050 \\
3.400 & 0.000 & -0.200 & 0.400 \\
3.450 & 0.000 & -0.200 & 0.250 \\
3.500 & 0.000 & -0.100 & 0.400 \\
3.550 & 0.000 & -0.200 & 0.150 \\
3.600 & 0.000 & -0.200 & -0.050 \\
3.650 & 0.000 & 0.150 & -0.250 \\
3.700 & 0.000 & 0.250 & -0.100 \\
3.750 & 0.000 & & 0.700 \\
3.800 & 0.000 & & 0.500 \\
3.950 & 0.000 & & 0.000 \\
\hline
\end{tabular}

projection beach with $10 \%$ slope and with the fortification located at its East vertex. This solution was proposed by LNEC - National Laboratory of Civil Engineering (Portugal). The main objectives of the studies described afterwards were:

- to analyze the validity of the solution proposed by LNEC with respect to the beach capacity feeding under the Summer agitation effect;

- to analyze and justify an alternative solution.

\subsection{Selection of the agitation and tide characteristics}

A group of hydrodynamic actions representative of the real conditions that the structure will be subjected to, have been selected. The following two waves and selected four tide plus wave cases were considered representative in terms of sedimentary dynamics:

Wave 1: height (offshore) $3.0 \mathrm{~m}$; period $12 \mathrm{~s}$; direction $225^{\circ}$;

Wave 2: height (offshore) $2.0 \mathrm{~m}$; period $8 \mathrm{~s}$; direction $287.5^{\circ}$.

\section{Selected cases:}

Case 1: $\max$ flood with wave 1;

Case 2: mid flood with wave 2;

Case 3: max ebb with wave 1;

Case 4: mid ebb with wave 2 .

In order to validate the physical model dynamics similarity adopted (LNEC), and for the analysis of different grain size diameters for the artificial beach feeding, other simulations were conducted. In the following, the first two cases are described, as well as a proposed solution and conclusions. 


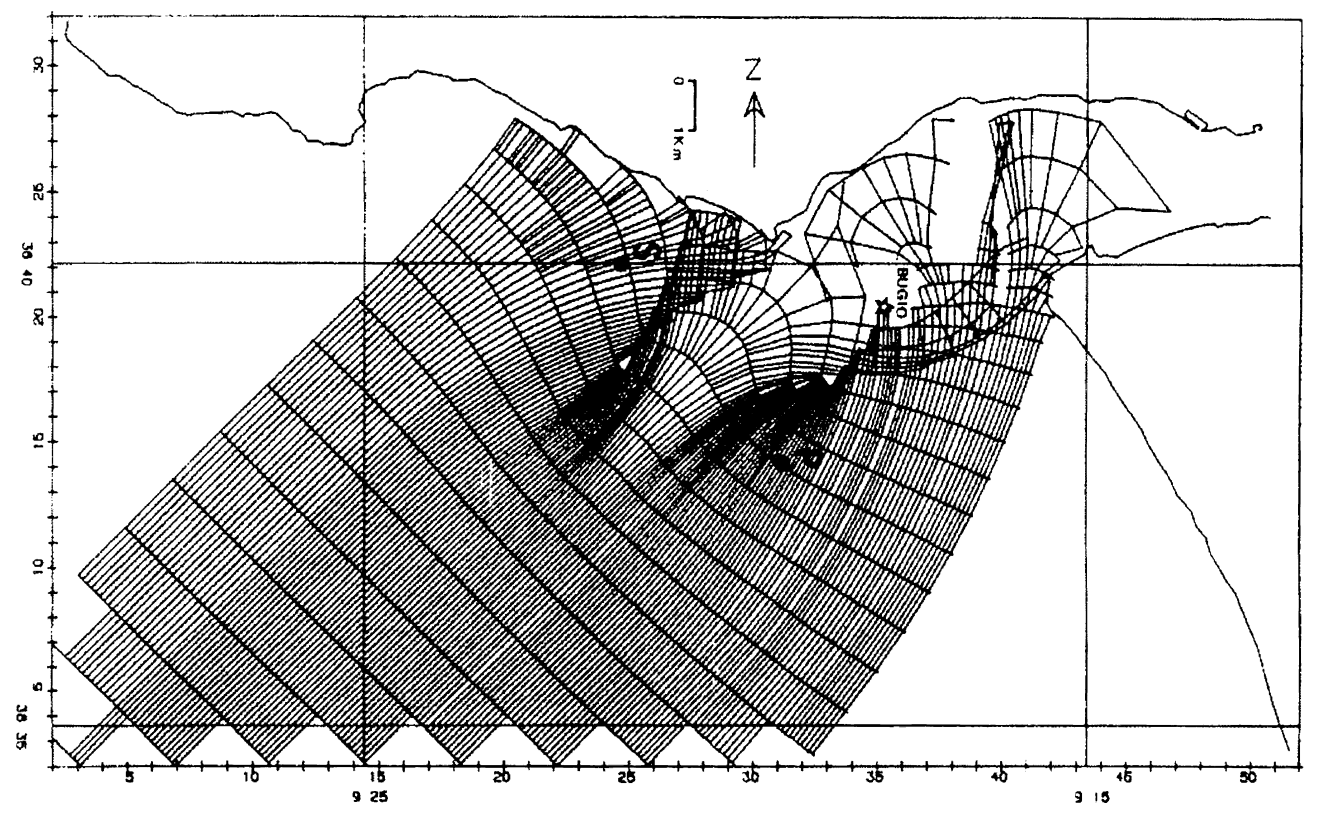

Fig. 3. Refraction plane corresponding to wave 1 (obtained by LNEC).

\subsection{Analysis of the two first cases}

\subsubsection{Analysis of Case 1}

The analysis of the refraction plane corresponding to wave 1 (Fig. 3) reveals a pronounced change in direction from off shore to the proximity of Bugio.

The following wave boundary characteristics for the nonlinear wave-current model were determined:

Wave height: $3.4 \mathrm{~m}$

Period: $12 \mathrm{~s}$

Direction: $180^{\circ}$

Current field calculated by Saint-Venant equations model reveals a maximum value of about $1.5 \mathrm{~m} / \mathrm{s}$, with a predominantly south-north direction. A time-dependent hydrodynamic characteristics of the joint action of the wave 1 over the high flow tide current field were obtained with resort to the wave-current extended Boussinesq model previously described.

Fig. 4 represents the water surface elevation $60 \mathrm{~s}$ after the beginning of the input wave boundary. Decomposition, refraction and diffraction figures are well represented, in general, as can be seen at the site and observed in the physical model.

Results of time-bed evolution are represented in Fig. 5. Sediment characteristics are approximately: $d_{50}=2.0$ and $d_{90}=3.0 \mathrm{~mm}$. In respect to zero initial bed values, the ones calculated $1 \mathrm{~h}$ after are varying from +1 to $-1 \mathrm{~m}$.

\subsubsection{Analysis of Case 2}

Refraction planes analysis corresponding to this situation reveals an instability of the orthogonal lines as a consequence of marked irregularities in the depth. We have decided to retain results up to point $S$ (Fig. 3), where the wave has the following characteristics:

Wave height: $1.5 \mathrm{~m}$

Period: $8 \mathrm{~s}$

Direction: $280^{\circ}$

Between point $\mathrm{S}$ and $\mathrm{S}$. Lourenço fortification, a Berkhoff's model has been utilized to propagate this signal wave as input boundary conditions. Wave height and phase results of this model are shown in Fig. 6.

As a result of this analysis, the following wave characteristics in the proximity of the Bugio lighthouse have been obtained:

Wave height: $0.8 \mathrm{~m}$

Period: $8 \mathrm{~s}$

Direction: $290^{\circ}$

Once more, the extended wave-current Boussinesq model was applied to obtain the time-dependent hydrodynamic characteristics of the joint action of wave 2 over the mid flow current field. Results at time $64 \mathrm{~s}$, corresponding to eight propagation wave periods, are shown in Fig. 7.

Results of time-bed evolution are represented in Fig. 8. The considered sediment characteristics were: $d_{50}=0.40$ and $d_{90}=0.60 \mathrm{~mm}$. As can be seen, sediment transport only has significance near the fortification and in the breaking zone, where these variations are in the order of $10 \mathrm{~cm}$. So, it is obvious from this situation that the associated hydrodynamic conditions do not guarantee the natural beach feeding. 


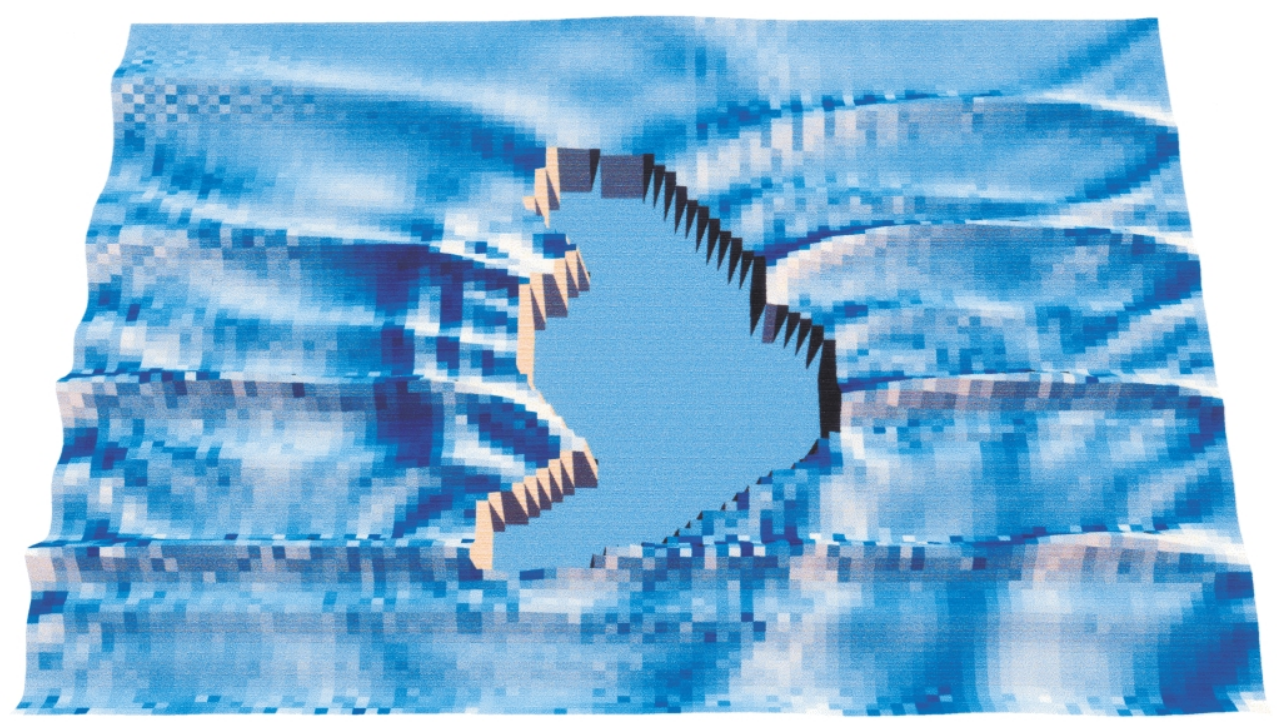

Fig. 4. Case 1: computed perspective view of the free surface elevation (five wave propagation periods after the wave action).

\subsection{Proposed solution}

As a general result of the studies conducted, for all cases presented in this section, both physical and mathematical approaches proved the inadequacy of the proposed solution. Therefore, the project evolved successively into: (1) a general increase of the breakwater height, (2) a spiral structure.

Finally, another proposed solution was tested; it is based on a circular dissipation platform with a level of $+2 \mathrm{~m}(\mathrm{ZH})$ (Fig. 9).

Fig. 10 shows a perspective view of the free surface elevation, in quasi-stabilized conditions. As can be seen, the wave action on the fortification was drastically reduced as a consequence of typical shallow-water phenomena, like reflection, refraction, diffraction and breaking on the dissipation platform constructed around the Bugio lighthouse.

\section{Conclusions}

A short to medium-term morphodynamic model was developed to predict the variation of bed topography in coastal regions.

Laboratory comparisons of measured and predicted bedlevel changes were performed. Although the laboratory conditions were generally very severe and theoretically out of range of applicability of this computational structure (high slopes, ripples formation and generation of vorticity) the simulated results showed reasonable agreement with the experimental ones.

A real-world application is described, and the main results and conclusions of the conducted studies are presented.

Results have shown that the computational structure presented is a valuable tool for studying the flow and the

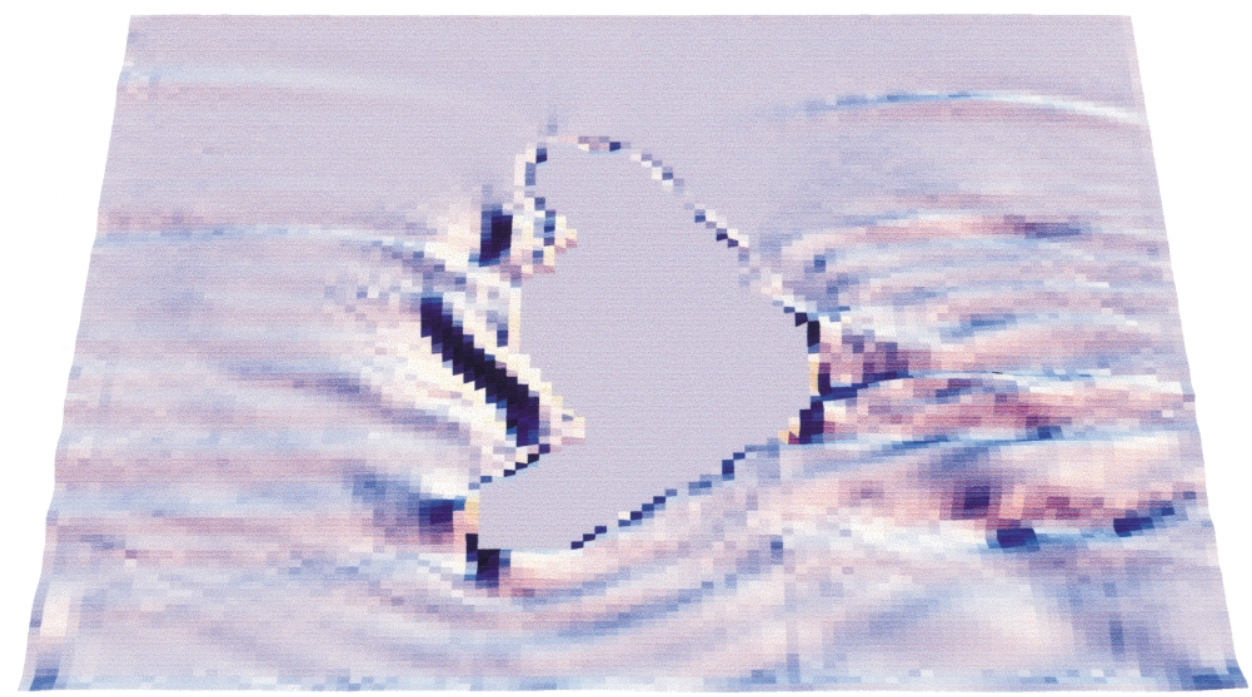

Fig. 5. Case 1: time-bed evolution. Computed bed changes $1 \mathrm{~h}$ after the current and wave action. 

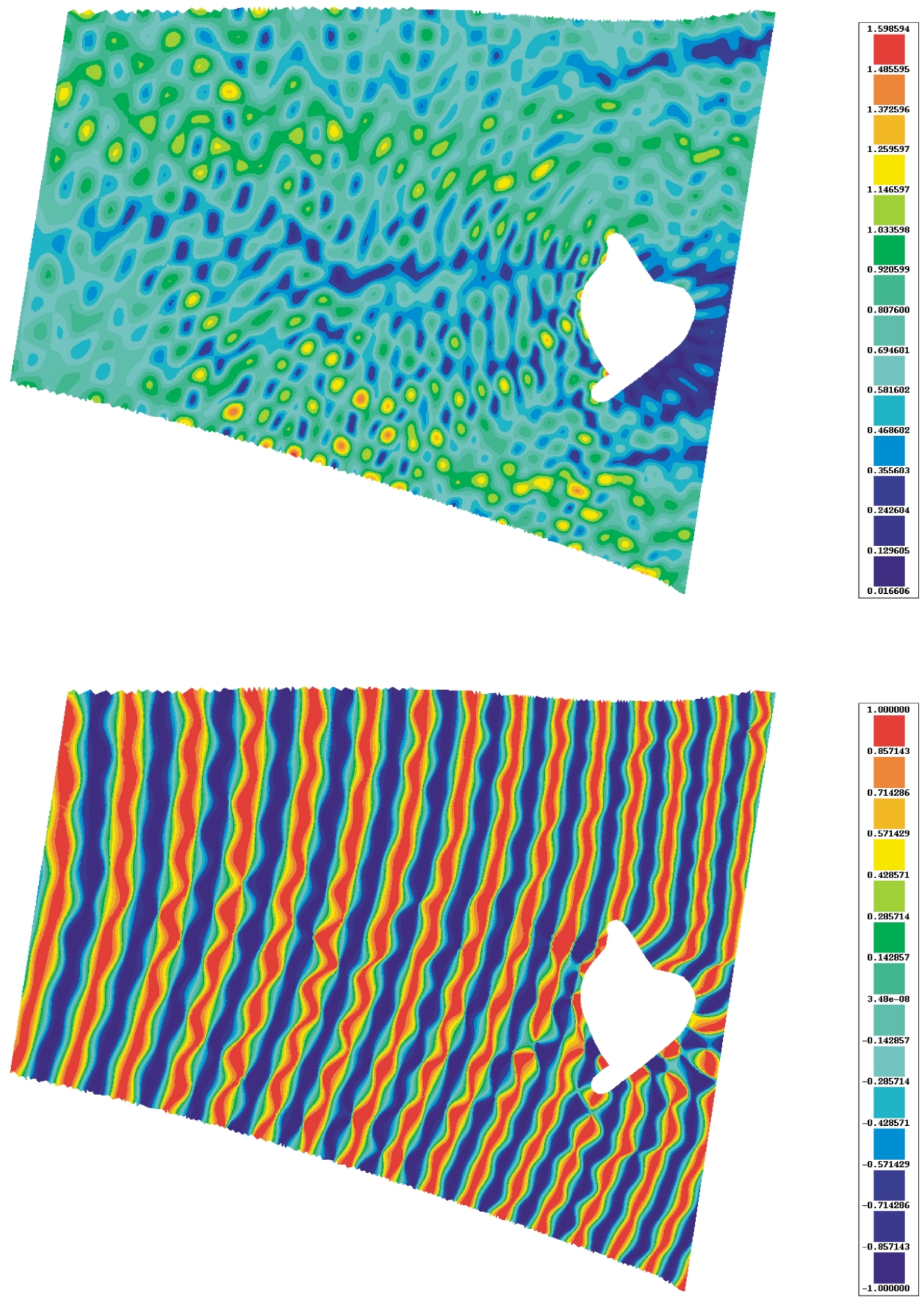

Fig. 6. Wave height (above) and phase results obtained by Berkhoff's model. 


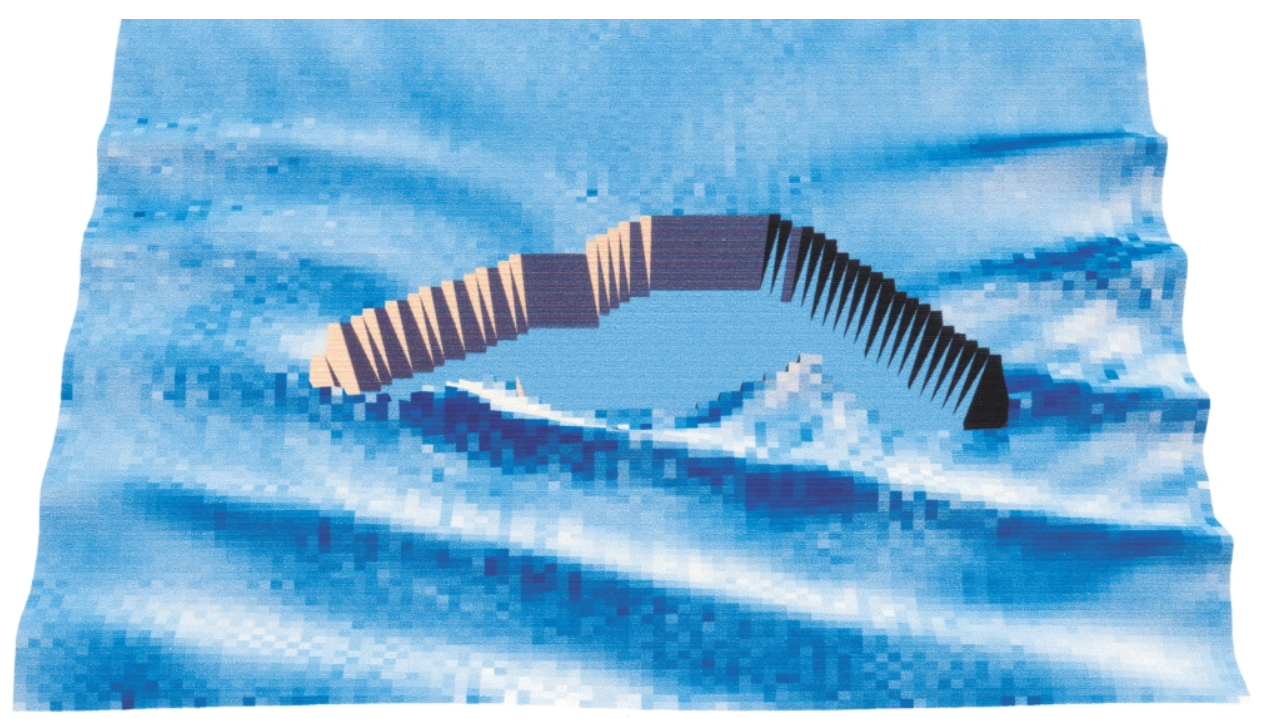

Fig. 7. Case 2: computed perspective view of the free surface elevation (eight wave propagation periods after the wave action).

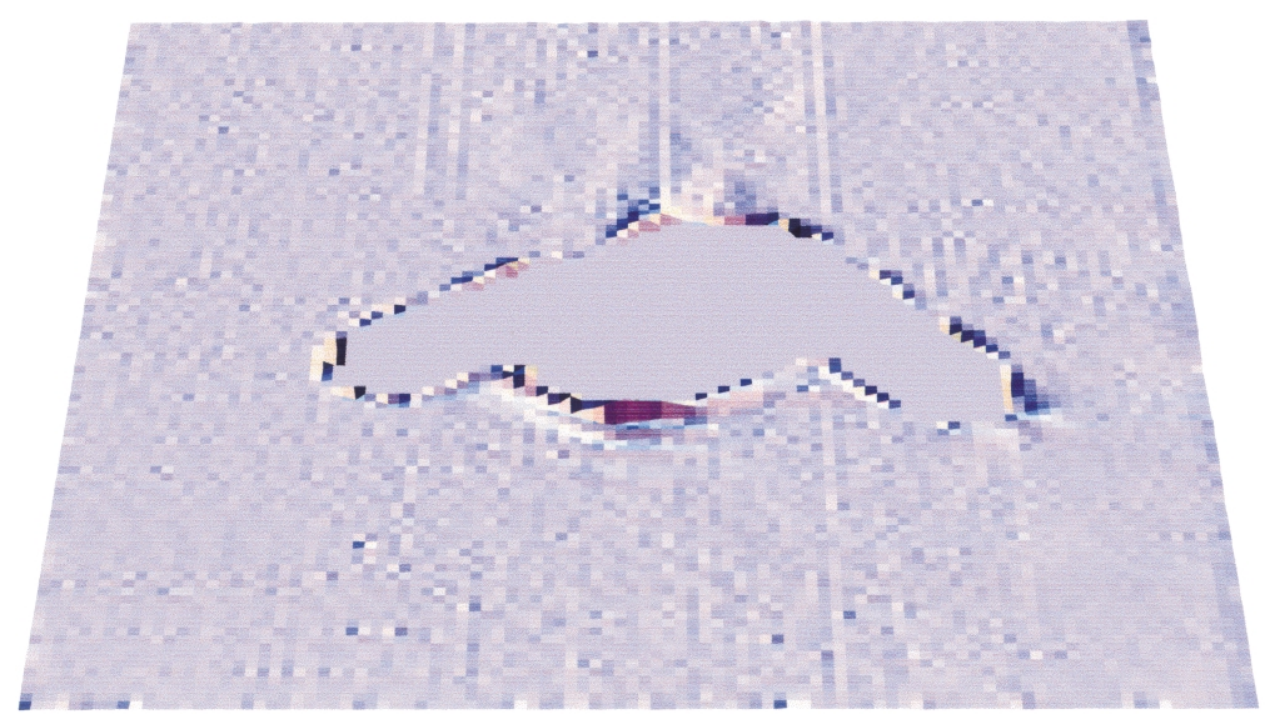

Fig. 8. Case 2: time-bed evolution. Computed bed changes $2 \mathrm{~h}$ after the current and wave action.

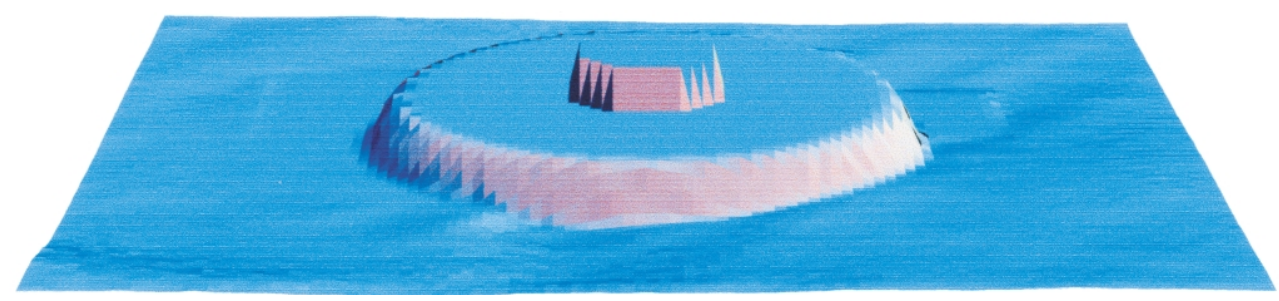

Fig. 9. Tested solution. Circular dissipation platform constructed around the fortification (with a level of $+2 \mathrm{~m}(\mathrm{ZH})$ and a base with about $150 \mathrm{~m}$ diameter). 


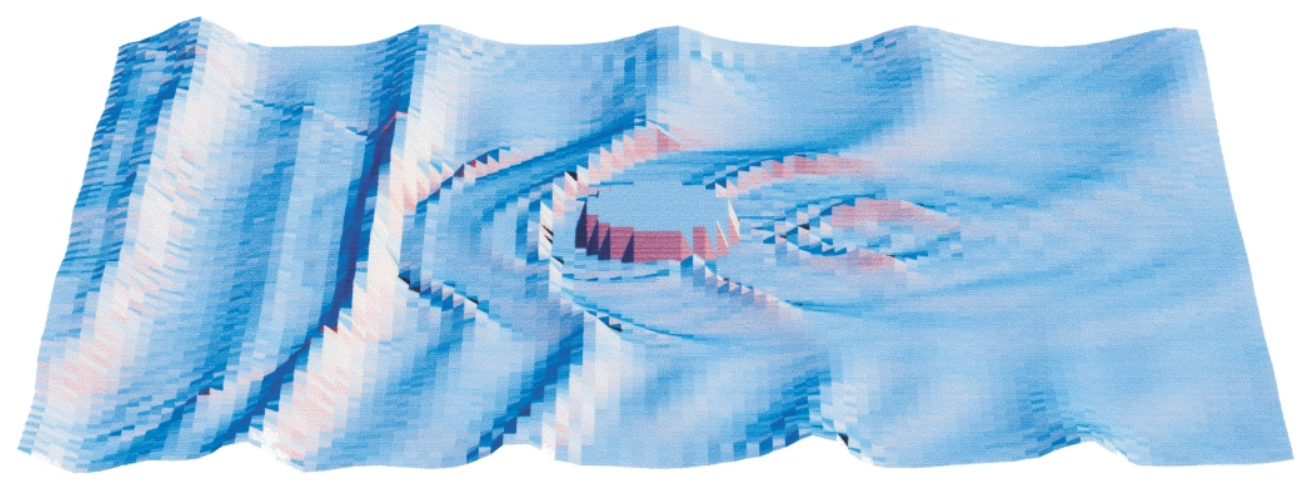

Fig. 10. Tested solution. Perspective view of the free surface elevation in quasi-stabilized conditions.

bed evolution in natural channels and coastal regions. However, it could be considered costly in terms of time nowadays, so only areas of a few thousands of elements may realistically be treated.

Anyway, the performance of the morphodynamic model in a real-life coastal behavior, considering this approach, is encouraging enough to proceed further research in order to extend this methodology to long-term analysis.

\section{Acknowledgements}

This work has been performed in the scope of the INDIA Project (INlet Dynamics Initiative: Algarve), which has been financed by EU, European Union, in the scope of The Marine Science and Technology (MAST-III) Programme.

\section{References}

[1] Grant WD, Madsen OS. Combined wave and current interaction with a rough bottom. J Geophys Res 1979;84(C4):1797-808.

[2] Asano T, Iwagaki Y. Proceedings of the 19th International Coastal Engng Conference, Houston, 1984. p. 2397-413.

[3] Dean RG, Dalrymple RA. Water wave mechanics for engineers and scientists. New Jersey: Prentice-Hall, Inc, 1984. ISBN 0-13-946038-1.

[4] Antunes do Carmo JS, Seabra-Santos FJ, Barthélemy E. Surface waves propagation in shallow-water: a finite element model. Int $\mathrm{J}$ Num Meth in Fluids 1993;16(6):447-59.

[5] Avilez-Valente P, Antunes do Carmo JS, Seabra-Santos FJ, Veloso-
Gomes F. General wave model for large coastal areas. Proceedings of Saint-Venant Symposium, Paris, 1997. p. 179-86.

[6] Longuet-Higgins MS, Stewart RW. Radiation stresses and mass transport in gravity waves with applications to surf-beats. J Fluid Mech 1962;13:481-504.

[7] Antunes do Carmo JS, Seabra-Santos FJ. On breaking waves and wave-current interaction in shallow-water: a $2 \mathrm{DH}$ finite element model. Int J Num Meth in Fluids 1996;22(5):429-44.

[8] Antunes do Carmo JS. Tsunamis: geração e riscos. Territorium, Revista de Geografia Física e Aplicada no Ordenamento do Território e Gestão de Riscos Naturais, Ed. Minerva. ISSN: 0872-8941, 7, 15 24 (in Portuguese), 2000.

[9] Antunes do Carmo JS. Contribuição para o Estudo dos Processos Morfodinâmicos em Regiões Costeiras e Esuarinas. PhD Thesis, University of Coimbra, Portugal (in Portuguese), 1995.

[10] Bagnold RA. Beach and nearshore processes. In: Hill MN, editor. The sea, vol. 3. New York: Interscience, 1963.

[11] Bagnold RA. An approach to the sediment transport problem from general physics. Geological Survey Professional Paper 422-42J, 1966.

[12] Bowen AJ. Simple models of nearshore sedimentation: beach profiles and longshore bars. In: McCann SB, editor. The coastline of Canada, Ottawa: Geological Survey of Canada, 1980. p. 1-11.

[13] Bailard JA, Inman DL. An energetics bedload model for a plane sloping beach: local transport. J Geophys Res 1981;86(C3):2035-43.

[14] Bailard JA. An energetics total load sediment transport model for a plane sloping beach. J Geophys Res 1981;86(C11):10938-54.

[15] Janssen CM, Ribberink JS. Grain-size influence on sand transport in oscillatory flow. ICCE'96 Proceedings, 1996.

[16] Silva PA, Antunes do Carmo J, Temperville A, Seabra-Santos F. Cross-shore beach profile predicted by two sand transport models. In: Blain WR, Brebbia CA, editors. Hydraulic Engineering Software VIII (Proceedings of Hydrosoft VIII). WIT Press, 2000. p. 207-15.

[17] Horikawa K. Nearshore dynamics and coastal processes. University of Tokyo Press, 1988 522p. 